\title{
HYPERTENSION
}

\section{The Symplicity of renal denervation}

Renal denervation offers an alternative

to drug therapy in patients with

treatment-resistant hypertension.

A new article published in The Lancet

reports that significant reductions in

systolic blood pressure (SBP) after

radiofrequency-mediated percutaneous

renal denervation are maintained for up

to 3 years post-procedure.

"This is a follow-on paper from our initial first-in-man report on the 12-month result published in 2009," explains Henry Krum of Monash University, Australia. Of the 111 patients from the Symplicity HTN-1 openlabel trial who agreed to be part of the follow-up study, 88 had complete data at 36 months. A $10 \mathrm{mmHg}$ drop in SBP occured in $69 \%$ of patients 1 month after the procedure, at 12 months this drop was seen in $85 \%$ and by the final 36 month follow-up point, $93 \%$ of patients had lower SBP. Three patients died, but the cause of death was unrelated to either procecure or denervation device. "The SBP responses were durable up to 3 years and the procedure was generally safe with minimal local aterial wall complications," clarifies Krum.

The Symplicity HTN-1 trial did not include a control group, and the authors recognize that a placebo effect might influence the results. However, patients with treatment-resistant hypertension normally have a worsening of blood pressure control, which did not occur in those who received renal denervation.

A larger trial is now planned, which has enrolled approximately 500 patients. "Our study was a proof-of-concept, the Simplicity HTN-3 study is the definitive trial, which will be hugely influential on clinical practice," added Krum.

Tim Geach

Original article Krum, H. et al. Percutaneous renal denervation in patients with treatment-resistant hypertension: final 3-year report of the Symplicity HTN-1 study. Lancet doi:10.1016/S0140-6736(13)61999-6 